\title{
Two-fluid plasma model for radial Langmuir probes as a converging nozzle with sonic choked flow, and sonic passage to supersonic flow
}

\author{
A. A. Howling, ${ }^{1} \mathrm{Ph}$. Guittienne, ${ }^{2}$ and I. Furno ${ }^{1}$ \\ ${ }^{1)}$ Ecole Polytechnique Fédérale de Lausanne (EPFL), Swiss Plasma Center, CH-1015 Lausanne, \\ Switzerland \\ ${ }^{2)}$ Helyssen, Route de la Louche 31, CH-1092 Belmont-sur-Lausanne, Switzerland
}

(Dated: 1 March 2019)

Using the Lambert function, Guittienne, Howling and Furno [Phys. Plasmas 25, 093519 (2018)] derived twofluid solutions for radial Langmuir probes in collisionless and isothermal plasma. In this Brief Communication, we point out the close analogy with classical compressible fluid dynamics, where the simultaneous flows of the ion and electron fluids experience equal and opposite electrostatic body forces in the inward radial flow of the plasma, which behaves as a converging nozzle. Hence, the assumed boundary condition of sonic flow of the repelled species at the probe is explained as choked flow. The sonic passage from subsonic to supersonic flow of the attracted species at the sonic radius is also interpreted using classical fluid dynamics. Moreover, the Lambert function can provide a general solution for one-dimensional, isothermal compressible fluids, with several applications.

Langmuir probes are one of the most fundamental diagnostics of plasma physics. ${ }^{1-4}$ A recent paper ${ }^{5}$ presented a mathematical treatment for radial Langmuir probes in collisionless, isothermal plasma by using the Lambert function. ${ }^{6}$ In this Brief Communication, it is shown that the Lambert function is in fact a general solution for onedimensional, isothermal compressible fluid equations in classical fluid dynamics. The analogy with fluid dynamics gives physical insight into the Lambert solution, and confirms the intuitive assumptions for the fluid boundary conditions in [5].

An analogy with classical fluid dynamics could be expected because [5] solves the Euler compressible fluid equations for mass continuity and momentum conservation, along with the ideal gas law, treating ions and electrons as two separate fluids. To illustrate this, Fig. 1(b) for radial probes in [5] is redrawn here in Fig. 1 using fluid dynamics terminology: The equivalence of the terms in classical fluid dynamics and in [5] is given in Table I, where the textbooks by Shapiro, ${ }^{7}$ and Landau and Lifshitz, ${ }^{8}$ are followed throughout. It can be seen that the flow towards the radial probe (cylindrical or spherical) is equivalent to one-dimensional (1D) radial flow in a converging nozzle. ${ }^{7}$ The 1D Cartesian case for plane probes $^{5,9}$ can be treated as flow in a straight tube, ${ }^{7}$ but is of less physical relevance to real Langmuir probes and is not considered further here.

The ions and electrons are collected at the probe's conducting surface, hence the probe is effectively a vacuum boundary for loss of the charged fluids. The neutrals which result from ion neutralization return to the neutral gas background, but do not interact with the ions or electrons in this collisionless approximation, and are not considered further. The ion and electron fluids both flow towards the probe according to the combined forces of their pressure gradient and the electric field; the resultant flow of the electron fluid is against the electrostatic force for the case shown in Fig. 1.

The properties of the non-perturbed equilibrium

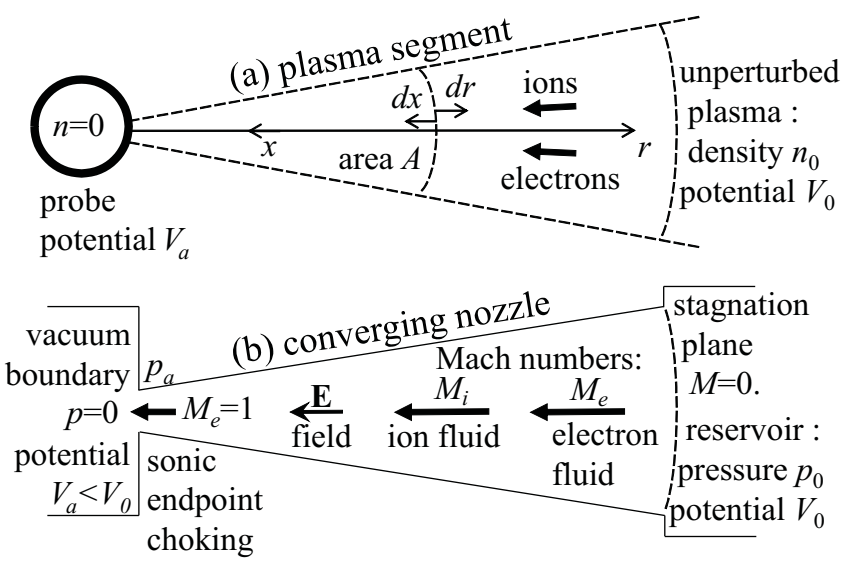

FIG. 1. (a) A cross-sectional segment of the cylindrical probe plasma geometry, showing the ion and electron flows, along $x$, from the unperturbed bulk plasma (equal densities $n_{0}$, plasma potential $\left.V_{0}\right)$ to the probe conducting surface, where they are collected $\left(n_{i}=n_{e}=0\right.$, probe potential $\left.V_{a}\right)$. (b) The same plasma segment represented as a converging nozzle for one-dimensional compressible flow ${ }^{7,8}$ of the ion and electron fluids with Mach number $M_{i}$ and $M_{e}$ respectively. The flows begin at the stagnation plane and end in an effective vacuum boundary caused by loss of the ion and electron fluids at the probe. The charged fluids experience an equal and opposite Coulomb body force in the electric field $\mathbf{E}$, shown for $V_{a}<$ $V_{0}$. For this case, the electron fluid undergoes sonic endpoint choking at the probe $\left(M_{e}=1\right)$, and the ion fluid accelerates in the electric field via sonic passage to supersonic speed.

plasma define a stagnation plane ${ }^{7}$ because the flow velocity towards the probe, from a large distance, tends to zero, by conservation of flux. ${ }^{5}$ Hence, the ion and electron fluids both enter the nozzle with subsonic velocity. There are no sidewalls, so no surface forces ${ }^{7}$ (i.e. no wall friction), and viscosity is neglected. The flow is assumed to be collisionless in this model, ${ }^{5}$ therefore the only external force acting on each flow is the electrostatic Coulomb body force which is equal and opposite for the ion and 


\begin{tabular}{|c|c|c|c|}
\hline Variable Name & Fluids $^{7,8}$ & Ref. 5 & Comment \\
\hline incremental dist. & $d x$ & $-d r$ & +ve along flow $^{-}$ \\
\hline pressure & $p$ & $n q T$ & $T$ in volts \\
\hline mass density & $\rho$ & $n m$ & particle mass $m$ \\
\hline mass flux density & $j=\rho u$ & $n m u$ & flow speed $u$ \\
\hline (sound speed) $^{2}$ & $c^{2}=p / \rho$ & $u_{\mathrm{th}}^{2}=q T / m$ & isothermal \\
\hline (Mach no.) $^{2}$ & $M^{2}$ & $U$ & $u^{2} / u_{\mathrm{th}}^{2}$ \\
\hline incremental area & $d A / A<0$ & $-d r / r$ & converging \\
\hline body force/volume & $F$ & $\pm n q E$ & electrostatic \\
\hline
\end{tabular}

TABLE I. Equivalence of one-dimensional compressible fluid dynamic variables ${ }^{7,8}$ with the variables of [5], which apply to the ion fluid and the electron fluid.

electron fluids. The electrostatic body force per unit volume is $\pm n q E=\mp n q \frac{d V}{d r}$, where the electric field $E$ is the negative gradient of the electric potential $V$. The electric field is calculated self-consistently using Poisson's equation. ${ }^{5}$ Body forces are usually neglected in compressible fluid dynamics because only gravity acts on neutral gases ${ }^{7}$ although an electromagnetic body force, due to plasma carrying current in a magnetic field, was introduced by Resler and Sears. ${ }^{10,11}$

The Euler equations for the electron and ion fluid flows in steady, 1D radial, isothermal and collisionless flow to a biased cylindrical probe are the conservation of mass, $2 \pi r n m u=$ const. , and the conservation of momentum, ${ }^{5}$

$$
\frac{1}{2} \frac{d U}{d r} \mp \frac{1}{T} \frac{d V}{d r}+\frac{d \ln (n)}{d r}=0,
$$

where $U=u^{2} / u_{\text {th }}^{2}$. In the term for $\frac{d V}{d r}$, the top sign (-) corresponds to electrons, and the bottom sign $(+)$ to ions. This equation, (28) in [5], was directly integrated, and by eliminating $n$ using mass conservation, the Lambert function solutions (33) were derived immediately.

However, for Euler equations generally, the momentum equation is not necessarily integrable, so the classical fluid dynamics solution proceeds via differential equations. ${ }^{7}$ Following that procedure here, the square of the mass conservation equation is first differentiated to give

$$
\frac{1}{r}+\frac{d \ln (n)}{d r}+\frac{1}{2 U} \frac{d U}{d r}=0
$$

then the differential term for $\ln (n)$ is eliminated from (1) to give

$$
-\frac{d U}{d r}=\frac{2 U}{1-U}\left(\frac{1}{r} \pm \frac{1}{T} \frac{d V}{d r}\right) .
$$

This is a new intermediate step for the solution in [5]. As before, the top sign (now + ) corresponds to electrons, and the bottom sign (now -) to ions.

In classical fluid dynamics, the Euler equations can be expressed as differential working equations, ${ }^{7}$ for example, for the dependence of the Mach number, $M$, on the duct area $A$ and retarding body force per unit volume $F$, as follows:

$$
\frac{d M^{2}}{d x}=\frac{2 M^{2}}{1-M^{2}}\left(-\frac{d \ln A}{d x}+\frac{F}{p}\right)
$$

which is greatly simplified in this case of isothermal flow. Isothermal flow effectively assumes infinite thermal conductivity because heat conduction via sidewalls is excluded here. Using Table I, it is clear that (3) is identical to the differential working equation (4). Hence the approach in [5] can be understood using classical fluid dynamics.

Equation (3) can be conveniently rearranged and integrated, thus rejoining the direct integration method in [5], to give:

$$
U-\ln U=\ln \left(r^{2}\right) \pm \frac{2 V}{T}+c s t,
$$

where $c s t$ is a constant of integration. In fluid dynamics textbooks, this is the point where analytical solutions end. ${ }^{7,11,13,14}$ However, raising (5) to the exponential and rearranging gives

$$
-U \mathrm{e}^{-U}=-\frac{1}{r^{2}} \mathrm{e}^{\mp \frac{2 V}{T}-c s t},
$$

which has the solution ${ }^{5}$

$$
U=-W\left[-\frac{1}{r^{2}} \mathrm{e}^{\mp \frac{2 V}{T}-c s t}\right],
$$

where $W$ denotes the Lambert function ${ }^{6}$ defined by $\alpha=$ $z \mathrm{e}^{z} \Leftrightarrow z=W[\alpha]$.

A more general solution can be found from the fluid equations: Provided that the only variables are the duct area $A$, and body forces $F(x)$ per unit volume, rearrangement and integration of (4) similarly yields

$$
M^{2}=-W\left[-\frac{1}{A^{2}} \mathrm{e}^{\int(2 F / p) d x}\right] .
$$

This shows that the Lambert function can be used to give a general solution to the Euler equations for 1D isothermal, compressible fluids.

The intermediate equation (3) and the differential working equation (4) can be exploited to justify the intuited boundary conditions in [5] as follows:

First, consider the special case of a field-free situation $(F$ and $\pm n q E=0)$ where the probe potential is maintained equal to the plasma potential, $V_{a}=V_{0}$, so there is no electric field to influence ions and electrons. Charged particles are neutralized on contact with the probe, which, therefore, is effectively a vacuum boundary for the flows. The resulting pressure gradients drive ion and electron flows from the equilibrium plasma to the probe sink. The boundary condition at the probe can be elucidated using fluid dynamics in two complementary ways:

1. The flows are initially subsonic because they enter from the stagnation plane, so the denominator $\left(1-M^{2}\right)$ in (4) is positive, as well as the numerator, $-\frac{d \ln A}{d x}=\frac{1}{r}$, for the converging nozzle. Therefore $\frac{d M^{2}}{d x}>0$, and the Mach number increases 
continually as the fluid flows towards the probe. The flow reaches a maximum limiting condition of sonic speed $M=1$ at the probe surface because, if $M^{2}>1$ were to occur, $\frac{d M^{2}}{d x}$ would change sign. ${ }^{7}$

2. The fluids empty into the probe vacuum boundary at the highest possible mass flow rate: From the mass flux density $j=\rho u$ and the momentum equation with $\frac{d V}{d r}=0$, a general result is that $\frac{d j}{d u}=\rho\left(1-M^{2}\right)$, hence the maximum mass flux density occurs at the sonic speed. ${ }^{8}$ Because the mass flow rate is conserved, this maximum in mass flux density must occur at the narrowest part of the converging nozzle, i.e. at the probe.

This sonic upper limit of flow speed and mass flux density at the exit of the converging nozzle is called sonic endpoint choking. ${ }^{15}$ The fluid pressure cannot drop to zero without the fluid accelerating to sonic velocity; a shock wave forms at the probe resulting in a pressure discontinuity from $p_{a}$ to zero (Fig. 1(b)). For isothermal conditions, the sonic speed is the same as the thermal velocity $u_{\text {th }}=\sqrt{\frac{q T}{m}}$, the root mean square velocity in any single direction. Hence the maximum flux density is $n_{a} u_{\mathrm{th}}$, where $n_{a}$ is the number density just before the collisionless shock at the probe surface. This sonic boundary condition was proven in [5], for planar probes, by requiring continuity between the branches of the Lambert function for repelled species $\left(W_{0}\right)$ and attracted species $\left(W_{-1}\right)$ in the limit $V_{a}=V_{0}$. Note that the flux density $n_{a} u_{\text {th }}$ in this fluid model is different from the classical kinetic theory flux density $\frac{1}{4} n_{a} \bar{v}=\frac{1}{\sqrt{2 \pi}} n_{a} u_{\mathrm{th}}$ (where $\bar{v}=\sqrt{\frac{8 q T}{\pi m}}$ is the mean thermal velocity), as discussed in [5].

Now consider the repelled species, for example, electrons when the probe voltage $V_{a}<V_{0}$ in Fig. 2(a). The numerator in (3) remains positive, hence the repelled species fluid always undergoes sonic endpoint choking. ${ }^{7}$ Consequently, the flux density of the repelled species at the probe is always $n_{a} u_{\mathrm{th}}$, which is consistent with the flux boundary condition intuited in [5]. The constant cst in (5)-(7), for a given probe voltage $V_{a}$, is therefore found by using $\{U, r, V\}=\left\{1, R_{p}, V_{a}\right\}$ for the boundary condition at the probe. The radial profile of the repelled species fluid velocity in Fig. 2 is the same as the subsonic curve in Fig. 8.7(c) of [7], and the solution for the square of the fluid velocity normalized to the sonic speed, for the repelled species, is:

$$
U=M^{2}=-W_{0}\left[-\frac{R_{p}^{2}}{r^{2}} \mathrm{e}^{\frac{2\left(V_{a}-V\right)}{T}-1}\right],
$$

where $W_{0}$ corresponds to the subsonic branch of the Lambert solution. ${ }^{5}$
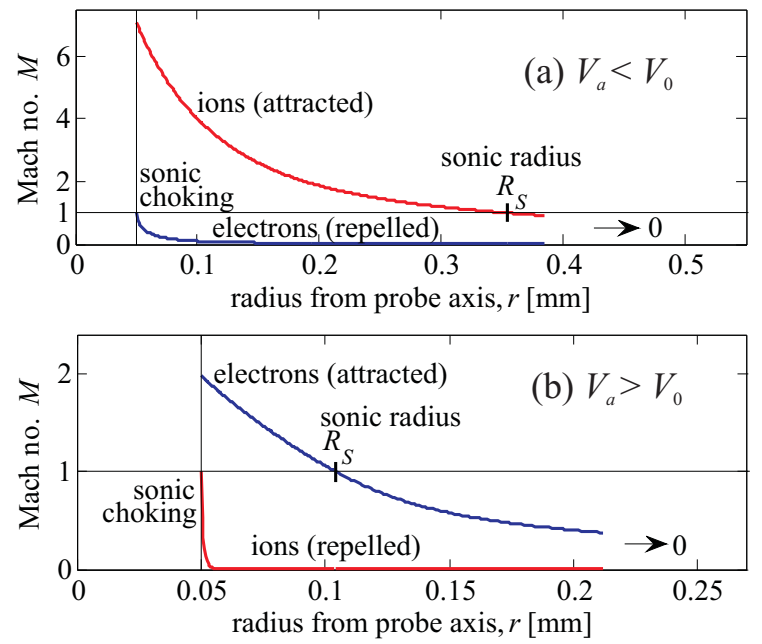

FIG. 2. Radial profiles of the fluid velocity for repelled and attracted species taken from Fig. 9 of [5]. Probe voltage (a) $V_{a}=15 \mathrm{~V}$, and (b) $V_{a}=25 \mathrm{~V}$. Probe radius $0.05 \mathrm{~mm}$ and plasma potential $V_{0}=20 \mathrm{~V}$. All velocities tend to zero at infinity.

Finally, consider the attracted species, for example, ions when the probe voltage $V_{a}<V_{0}$ in Fig. 2(a). Starting from the field-free case $V_{a}=V_{0}$, the ions will also experience sonic endpoint choking at the probe. ${ }^{5}$ However, as the probe voltage is lowered, there comes a point when the numerator of (3) becomes zero at $R_{p}$ when $\left[\frac{d V}{d r}\right]_{R_{p}}=\frac{T}{R_{p}}$. For all probe voltages below this value $V_{S}$, there is a critical radius within the plasma where the numerator and denominator are both zero and simultaneously change sign, so that $\frac{d M^{2}}{d x}$ remains positive. Hence the attracted species fluid continues to accelerate, in the electric field, to supersonic speeds as the fluid approaches the probe. This is called sonic passage. ${ }^{7}$ At this critical radius, $M=1$ and $\left[\frac{d V}{d r}\right]_{R_{S}}=\frac{T}{R_{S}}$, where $R_{S}$ is the sonic radius, as shown in Fig. 2. The radial profile of the attracted species fluid velocity is the same as the critical curve in Fig. 8.7(e) of [7]. The constant cst in (5)-(7) is found using $\{U, r, V\}=\left\{1, R_{S}, V_{S}\right\}$, so that the solution for the square of the fluid velocity normalized to the sonic speed, for the attracted species, is:

$$
U=M^{2}=-W_{k}\left[-\frac{R_{S}^{2}}{r^{2}} \mathrm{e}^{\frac{-2\left(V_{S}-V\right)}{T}-1}\right],
$$

where $k=0$ corresponds to the subsonic branch of the Lambert solution, and $k=-1$ corresponds to the supersonic branch, with continuity at the sonic radius in Fig. 2.

Thus, the solutions for the repelled and attracted species, (37) in [5], are self-consistent with a classical fluid dynamic treatment, (9) and (10). Unfortunately, the fluid dynamics approach does not appear to give more information about the sonic passage than already deduced in Fig. 8 and Appendix B.1 of [5]. Therefore, 
the sonic radius $R_{S}$ and sonic potential $V_{S}$ remain as the final parameters requiring numerical solution, involving Poisson's equation. ${ }^{5}$

Previous examples of choked flow for 1D Euler equations describing plasma, include single-fluid channel flow for MHD generators and plasma accelerators, ${ }^{10,11}$ and electron flow in field-effect transistors. ${ }^{16}$ The Parker model for the solar wind involves single-fluid isothermal flow, where diverging-nozzle expansion is retarded by the sun's gravity; this flow also undergoes sonic passage to supersonic expansion. ${ }^{13}$ Collisionless bow shock occurs against planetary magnetospheres, analogous to collisionless shock at the probe surface in Fig. 1. The analytical expression for the gravitational force means that the fluid equations (4) and (8) for the solar wind model have an exact Lambert function solution. ${ }^{17}$

To summarize, isothermal collisionless plasma surrounding a radial Langmuir probe has been treated as a converging nozzle for ion and electron fluids affected by electrostatic body forces: The repelled fluid undergoes sonic endpoint choking at the probe surface; the attracted fluid undergoes sonic passage at the sonic radius. This fluid dynamic approach is consistent with the boundary conditions in [5], and provides physical insight into the Lambert function solution. Furthermore, this function can provide a general solution for $1 \mathrm{D}$, isothermal compressible fluids, with additional applications in plasma physics ${ }^{18}$ such as solar wind and channel flow, as well as Langmuir probes.
${ }^{1}$ H. M. Mott-Smith and I. Langmuir, Phys. Rev. 28, 727 (1926).

${ }^{2}$ L. Tonks and I. Langmuir, Phys. Rev. 34, 876 (1929).

${ }^{3}$ F. F. Chen, Electric Probes (in "Plasma Diagnostic Techniques", ed. R. H. Huddlestone and S. L. Leonard, Academic Press, New York, 1965) Chap. 4, pp. 113-200.

${ }^{4}$ J. E. Allen, Plasma Sources Sci. Technol. 18, 014004 (2009).

${ }^{5}$ P. Guittienne, A. A. Howling, and I. Furno, Phys. Plasmas 25, 093519 (2018).

${ }^{6}$ R. M. Corless, G. H. Gonnet, D. E. G. Hare, D. J. Jeffrey, and D. E. Knuth, Adv. Comput. Math. 5, 329 (1996).

${ }^{7}$ A. H. Shapiro, The Dynamics and Thermodynamics of Compressible Fluid Flow, Volume I (The Ronald Press Company, New York, 1953).

${ }^{8}$ L. D. Landau and E. M. Lifshitz, Fluid Mechanics, Vol. 6 of "Course of Theoretical Physics" (Pergamon Press, 1966).

${ }^{9}$ B. A. Alterkop, I. D. Dubinova, and A. E. Dubinov, J. Exp. Theor. Phys. 102, 173 (2006).

${ }^{10}$ E. L. Resler and W. R. Sears, J. Aeronaut. Sci. 25, 235 (1958).

${ }^{11}$ T. M. York and H.-B. Tang, Introduction to Plasmas and Plasma Dynamics (Elsevier Inc. Academic Press, London, 2015).

${ }^{12}$ M. A. Lieberman and A. J. Lichtenberg, Principles of Plasma Discharges and Materials Processing (John Wiley and Sons, 2nd ed., Hoboken, New Jersey, 2005).

${ }^{13}$ G. K. Parks, Physics of Space Plasmas (Addison-Wesley Publishing Company, Redwood City, CA, 1991).

${ }^{14}$ B. B. Rossi and S. Olbert, Introduction to the Physics of Space (McGraw Hill Book Co., New York, 1970).

${ }^{15}$ T. Walters, Chem. Eng. 107, 70 (2000).

${ }^{16}$ M. I. Dyakonov and M. S. Shur, Phys. Rev. B 51, 14341 (1995).

${ }^{17}$ A.-M. Piso, (MIT) "The Solar Wind" http://demonstrations.wolfram.com/TheSolarWind/, Wolfram Demonstrations Project (2011).

${ }^{18}$ I. D. Dubinova, Plasma Phys. Reports 30, 872 (2004). 